\title{
Period-decoupled Short-term Price Prediction Model Based on Artificial Neural Network and Least Squares-Support Vector Machine Approach Optimized by Bacterial Colony Chemotaxis Algorithm
}

\section{Song Xue ${ }^{1}$}

State Grid Energy Research Institute, Changping District, Beijing 102209, China

E-mail: xuesongbjhde163. com

\section{Shangdong Yang}

State Grid Energy Research Institute, Changping District, Beijing 102209, China

E-mail:yangshangdongesgeri.sgcc.com.cn

\section{Xiaoxuan Zhang}

State Grid Energy Research Institute, Changping District, Beijing 102209, China

E-mail:zhangxiaoxuan@sgeri.sgcc.com.cn

The short-term price prediction result is an important bidding strategy basis for generating the enterprise consumers. Firstly, the index system for factors affecting the short-term electricity price was constructed and the core influencing factors were selected based on Artificial Neural Network (ANN); then, Bacterial Colony Chemotaxis (BCC) algorithm was built to determine the extra-parameters used in the least squares-support vector machine (LS-SVM) for short-term electricity price forecasting. In order to avoid BCC trapped in the local optimum, a perturbation was applied to the population to extricate itself out of the local optimal and start a new search and optimized BCC algorithm.

\footnotetext{
${ }^{1}$ Speaker

Correspongding Author
} 


\section{Introduction}

The short-term price prediction result is an important bidding strategy basis for generating enterprise consumers in the day-ahead market, which features the practical significance. Currently, the time-series method and the artificial intelligence method have been widely used in the short-term price prediction. While the time-series method is predicted from its own rule without considering influence of the market factors on short price, the artificial neural network method exists certaindiscreteness and will be easy to fall into local optimum trap.

Some short-term electricity price methods have been proved to be easy to fall into local optimum[1-2], and the prediction accuracy had to be improved; besides, many researches failed to quantify the bidding strategies of power generation enterprises[3-5], which affected the shortterm electricity price.

As the short-term price is also affected by seasons, climate and other factors, which feature significant periodicity, and the characteristics of different periods of the price change dramatically, there is a huge difference between the price change characteristics of different periods. In order to overcome disadvantages of the traditional prediction, the core influencing factors which were selected based on Artificial Neural Network (ANN) and BCC-LS-SVM method for short-term electricity price forecasting were constructed in order to avoid BCC as trapped in local optimum; moreover, a perturbation was applied to the population to optimize $\mathrm{BCC}$ algorithm, which can effectively improve the prediction accuracy and convergence speed.

\section{Influencing Factors Selection based on ANN}

The chronological price sequence highlights higher uncertainty, and it's difficult to accurately predict the short-term price with single traditional prediction method. According to different time points, the price sequence can be divided into 24 electricity price series (i.e. period-decoupled price series, if the market is clearing every half an hour, there are 48 electricity price series), and the price changes is relatively stable for each point in the time sequence's historical data [1]; in this sense, the price of each period-decoupled was respectively predicted in this paper. Factors affecting the short-term electricity price can be divided into three categories: the generators' bidding strategy factors, the external environmental factors and the system parameters- influenced factors. Usually, these data are issued by the trading organizations and the scheduling department of electricity market to market entities. The index system is shown in Fig.1.

In the index system, the system available capacity is the difference between the system capacity and the planned maintenance capacity. The system load rate is the ratio for system load and available capacity, which can reflect the market supply and demand situation [2].

ANN (Artificial Neural Network) is a number of artificial neurons connected with each other simply as applied to simulate the biology neural network, and then obtain information from outer environment or other neural. After simple calculations, the outputs are transported back to the outer environment or other neural. BP (Back Propagation) Error Back propagation learning algorithm with two processes: the forward propagation of information and the backward propagation of error, which is the most representative and widely used in ANN; thus we choose BP to find out the key factors for prediction.

With a random set of weights and thresholds, BP algorithm firstly selects a sample as input to calculate the output value with feed-forward method; then change the weights and thresholds with back-propagation model and get a new set of weights and thresholds. As a cycle, all samples are calculated. Repeat this cycle until the error is less than the specified accuracy. With increase of the number of iterations, the system error will decrease and finally converge to a stable set of weights and thresholds to help us get the influencing factors of coal price. The specific calculation process has been developed ${ }^{[6]}$. The core influencing factors can be selected by ANN. 


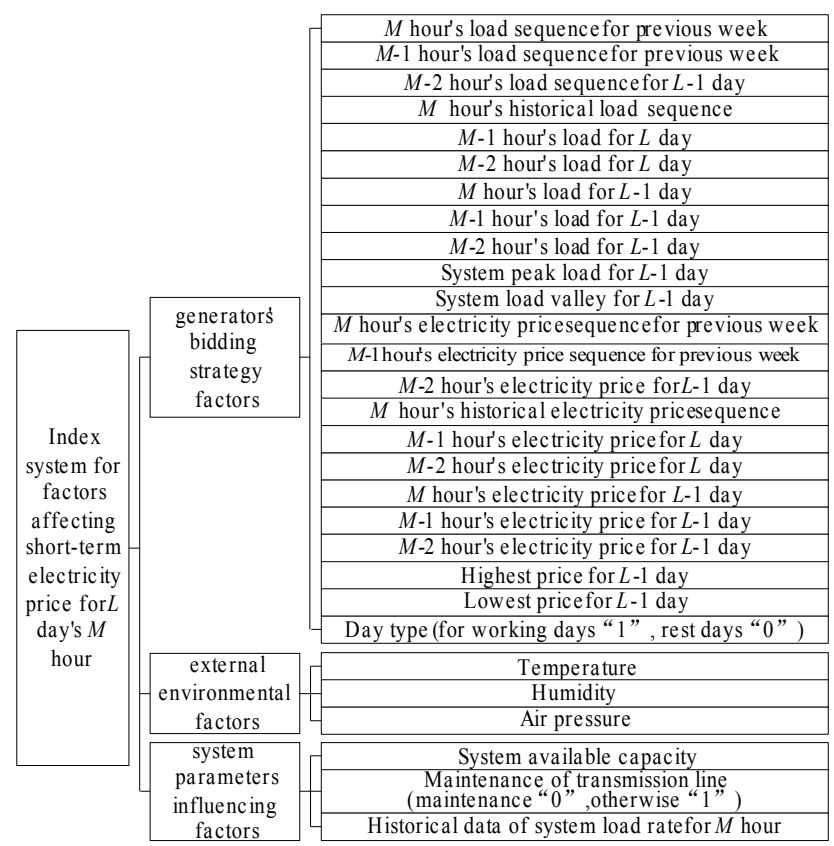

Figure 1 : Index System for Short-term Electricity Price-affected Factors

\section{Short-term Electricity Price Forecasting Model based on Optimized BCC-LS- SVM Method}

\subsection{Model of Least Squares-Support Vector Machine}

LS-SVM can solve small sample, non-linear, high dimension and local minima problems, and has been successfully applied to classification, function approximation and time series prediction. Concrete calculation process has been developed[7].

Support vector machine (SVM) is specialized in solving actual problems characterized by small samples, non-linear features, high dimensions and local minimum values. It has been successfully applied to deal with categories, function approaching and time series forecasting, etc. Least Squares-Support Vector Machine (LS-SVM) proposed by Suykens is viewed as the improvement of standard SVM with a lot of advantages: the non-equation constraints in standard SVM are replaced by equitable ones and quadratic programming problem is converted to solve the linear equations directly. Given a set $\left\{\boldsymbol{x}_{i}, \boldsymbol{y}_{i}\right\}_{i=1}^{N}$, where $\boldsymbol{x}_{i} \in \boldsymbol{F}^{N}$, is the inputs and $\boldsymbol{y}_{\boldsymbol{i}} \in \boldsymbol{F}^{\boldsymbol{N}}$ is the corresponding outputs. The non-linear function $\boldsymbol{f}(\bullet)$ is aiming at converting the samples to the characteristics space. The model of LS-SVM can be described as follows:

$$
h\left(a_{i}\right)=G^{T} f\left(x_{i}\right)+m
$$

Where, $\boldsymbol{G}$ and $\boldsymbol{m}$ are parameters to be examined. $\boldsymbol{G}$ and $\boldsymbol{m}$ can be obtained by minimizing the following objective function.

$$
F=0.5\|\boldsymbol{G}\|^{2}+\zeta \cdot F_{1}
$$

Where, $\zeta$ is regulation factor; $F_{1}$ is loss function. The optimization problem can be

$$
\left\{\begin{array}{l}
\min Z\left(G, d_{i}\right)=0.5\|G\|^{2}+0.5 \zeta \sum_{i=1}^{N} d_{i}{ }^{2} \\
\text { s.t. } y_{i}=G^{T} f\left(x_{i}\right)+m+d_{i}, i=1,2, \ldots, N
\end{array}\right.
$$

described as:

The Lagrange function of

$$
L\left(\boldsymbol{G}, \lambda_{i}, \boldsymbol{m}, \boldsymbol{d}_{i}\right)=Z+\sum_{i=1}^{N} \lambda_{i}\left[\boldsymbol{y}_{i}-\boldsymbol{G}^{T} \boldsymbol{f}\left(\boldsymbol{a}_{\boldsymbol{i}}\right)-\boldsymbol{m}-\boldsymbol{d}_{i}\right]
$$


Where, $\lambda_{i} \geq 0$, is the Lagrange mutiplier; $\boldsymbol{d}_{i}$ is the error. According to the Karush-KuhnTucker conditions, the following equations can be obtained.

$$
\partial \boldsymbol{L} / \partial \boldsymbol{G}=0, \partial \boldsymbol{L} / \partial \mathrm{h}_{i}=0, \partial \boldsymbol{L} / \partial \boldsymbol{m}=0, \partial \boldsymbol{L} / \partial \boldsymbol{d}_{i}=0
$$

Then,

$$
G=\sum_{i=1}^{N} \lambda_{i} f\left(x_{i}\right), \quad \sum_{i=1}^{N} \lambda_{i}=0, \lambda=\zeta d_{i}
$$

$\boldsymbol{y}_{i}-G^{T} f\left(x_{i}\right)-m-d_{i}=0$

The linear equations are obtained after eliminating $\boldsymbol{G}$ and $\boldsymbol{d}_{\boldsymbol{i}}$.

$$
\left[\begin{array}{cccc}
0 & 1 & L & 1 \\
1 & k\left(x_{1}, x_{1}\right)+\frac{1}{r} & L & k\left(x_{1}, x_{N}\right) \\
M & M & M & M \\
1 & k\left(x_{N}, x_{1}\right) & L & k\left(x_{N}, x_{N}\right)+\frac{1}{r}
\end{array}\right]\left[\begin{array}{c}
m \\
\lambda_{1} \\
M \\
\lambda_{N}
\end{array}\right]=\left[\begin{array}{c}
0 \\
y_{1} \\
M \\
y_{N}
\end{array}\right]
$$

$\lambda_{i}$ and $m$ can be obtained by calculating these linear equations. The model associated with inputs $\boldsymbol{x}$ is described as:

$$
\hat{y}(x)=\sum_{i=1}^{N} \lambda_{i} \varphi\left(x_{i}, x\right)+m
$$

Where, $\varphi\left(\boldsymbol{x}_{i}, \boldsymbol{x}\right)=\boldsymbol{f}\left(\boldsymbol{x}_{\boldsymbol{i}}\right)^{T} \boldsymbol{f}(\boldsymbol{x})$ is a kernel function subject to Phil Mercer theorem. In this paper, RBF kernel function is adopted: $\varphi\left(\boldsymbol{x}, \boldsymbol{x}_{\boldsymbol{k}}\right)=\exp \left(-\left\|\boldsymbol{x}-\boldsymbol{x}_{k}\right\|^{2} / 2 \mu^{2}\right)$.

It can be seen that kernel parameter $\mu$ and regulation factor $\zeta$ in (3.2) have a large impact on the accuracy of LS-SVM model. These two parameters can be obtained in the training period of RNM-BCC optimization algorithm.

\subsection{Parameters Optimization with BCC}

Bacterial colony chemotaxis (BCC) algorithm, which is developed from the bacterial chemotaxis (BC) algorithm, is a swarm intelligent algorithm that can realize the information communication among individuals. Bacterial colony can constantly feel the change in their environment through the individual bacteria constant moving and use their previous experience to find the global optimal point. BCC algorithm highlights better robustness. Steps of BCC algorithm are described as follows [8]:

Step1: set the population size of the bacterial colony and initialize the position of individual bacterium and the sense limit.

Step2: calculate the objective function(see Equation (3.2)) of the individuals under the initial conditions; determine and record the optimal solution.

Step3: at step $k$, the bacteria $j$ acquires information about its environment and find the centre location of other bacteria colony. The centre location can be assigned a better objective function value in the perception limitation. The centre location can be expressed as:

$$
\hat{\beta}(j)=\omega k\left(a_{j, k}, \hat{a}_{j, k}\right)
$$

Where $\hat{a}_{j, k}$ denotes the centre bacteria; $k\left(a_{j, k}, \hat{a}_{j, k}\right)$ is the distance between bacteria $j$ and the centre location $\hat{\beta}(j) ; \omega$ is a random number between $(0,1)$.

Step4: according to individual bacterial chemotaxis (BC) algorithm, the bacteria $j$ can get to a new location $\psi(j)$.

$$
\psi(j)=\psi^{\prime}(j)+p(j)
$$

Where $\psi^{\prime}(j)$ is the current location of bacteria $j ; p(j)$ is the expectation of the next location.

$$
p(j)=v t \mathrm{e}^{i \theta}
$$

Where $v, t$ and $\theta$ denote movement speed, time and rotation angle respectively..

Step5: compare the objective function value at location $\hat{\beta}(j)$ and $\psi(j)$. The bacteria $j$ will move to location of higher function value at Step $k+1$,

Step6: in order to avoid abandoning the original better location due to randomness of the algorithm, wheneve the bacterial colony moves, the bacteria at wore location will move to the best location to improve the performance of the algorithm. 
Step7: update the best location and relative parameters. Repeat Steps 3 to 6 until the end condition is met, then exit the loop.

It should be noted that there should be two key factors in the selection of hyper-parameters based on BCC: 1) how to describe the location of bacteria by hyper-parameters; which will directly affect the searching efficiency and convergence speed; 2) how to define the fitness function.

In the proposed algorithm, every bacterial colony is required to provide one potential solution, which is called hyper-parameters combination. Here, a hyper-parameters combination is defined as one-dimensional combined vector $x$, for example, RBF: $\boldsymbol{x}=(\zeta \mu)$. In this research, set $\boldsymbol{x}=\left(\log _{2} \zeta \log _{2} \mu\right)$ because this method has a higher searching efficiency and has got more stable optimization result.

In this problem, in the course of calculating the fitness value of bacterial colonies, the fitness function can guide BCC algorithm to move to a better solution. It shows that it should be defined for different problems. The higher the fitness value is, the better the location will be. The fitness function is defined as follows:

$$
G=-\frac{1}{N}{ }_{j=1}^{N} \frac{\left|c_{j}-c_{j}\right|}{c_{j}} 100
$$

Where $c_{j}^{\prime}$ and $c_{j}$ denote the training results and the actual results respectively.

In order to solve the problem prone to be trapped in the local optimum of BCC, we perturb the population. It is difficult to get rid of the situation that the best individual of the current generation is the local optimum by using the BCC to analyze. When the best individual does not change in successive generations, the algorithm is trapped in local optimum; in this sense, a perturbation should be applied to the population to extricate itself out of the local optimal and start a new search. The complete ANN and LS-SVM method optimized by perturbation BCC flowchart is shown in Fig. 2:

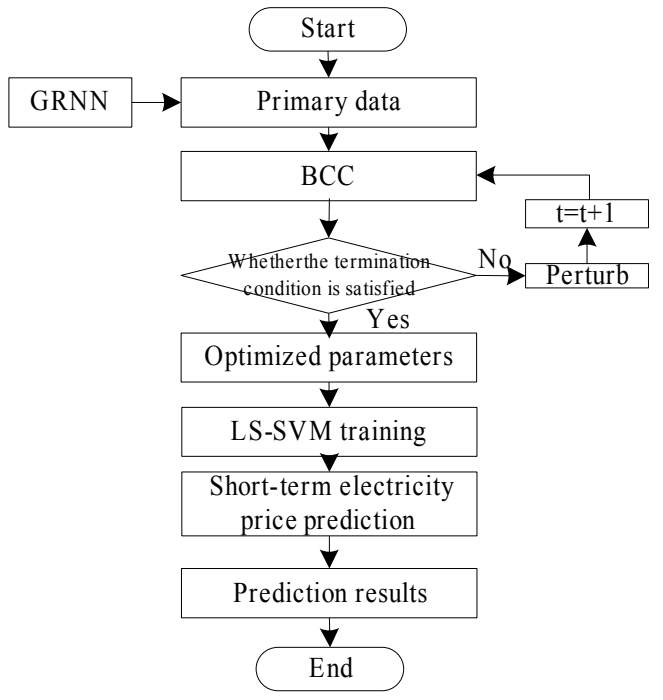

Figure 2 : Flow chart of ANN and Optimized BCC-LS-SVM

Step1: establish the regression function.

The optimal regression function of LS-SVM is established according to the non-linear function. The inputs of SVM are the key factors influencing the short-term electricity price as obtained by ANN while the output is the vehicle ownership.

Step2: determine the kernel function and parameters.

Radial Basis Function (RBF) kernel function is adopted in this paper and regulation factor and kernel parameters are obtained by using BCC method. Conditions needed to be satisfied that probability amplitude of $\mathrm{T}$ generation and $\mathrm{T}+1$ generation is consistent.

Step3: establish the prediction model of short-term electricity price. 
The regression optimization problems and constraints of LS-SVM are firstly created. The optimal coefficients of the regression function are obtained by using the Lagrange function; then get the short-term electricity price prediction model.

Step4: prediction.

The model is actually a black box model. The influencing factors of long-term electricity price are the inputs and the long-term electricity price is obtained by the simulation learning.

\section{Conclusion}

Accurate short-term price is significant in China, especially when the reform of the electricity market is considered in the near future. The main innovation lies in the forecasting method of short-term electricity price in this paper and is expected to achieve an ideal forecasting result.

\section{References}

[1] Q. C. Duan, M. Zhao, D. X. Wang. Short-term electricity price forecasting based on hybrid particle swarm optimization and normalized radial basis function neural network[J]. Power System Protection and Control, 2009, 37(18): 38-42.

[2] X. Zhang, X.-F. Wang, F.-.H. Chen, Y. E Bin, H.Y. Chen. Short-Term Electricity Price Forecasting Based On Period-Decoupled Price Sequence[J]. Proceedings of the CSEE, 2005, 25(15): 1-6.

[3] P. Yang, D.H. You, P.-.Y. Xie. Price Forecasting Based on Genetic Algorithm and BP Neural Network[J]. Water Resources and Power, 2003, 21(2): 84-86.

[4] F. J. Nogales, J. Contreras, A. J. Conejo, Espinola R.. Forecasting Next Day Electricity Prices by Time Series Models[J].IEEE Trans on Power Systems, 2001, 17(2):342-348.

[5] H. Y. Yamin, S. M. Shahidehpour, Z. Li, Adaptative Short-term Electricity Price Forecasting Using Artificial Neural Networks in the Restructured Power Markets[J].Electr Power Energy Syst, 2004, 26: 571-581.

[6] Q. Ying, Y. Tan, P. Chi, ANN Based Power Engineering Cost Forecas[J]. Journal of Jiangxi Vocational and Technical College of Electricity, 2011, 24: 21-23.

[7] M. Zeng, Y. Wang, S. Xue, Z. j. Wang. Prediction of China's Coal Price During Twelfth Fiveyear-plan Period Based on ANN and RNM-BCC-LS-SVM Method[J]. Journal of Applied Sciences, 2013, 13(15): 3055-3060

[8] M. Zheng, C. Q. LÜ , K. Tian, S. Xue. Least Squares-support Vector Machine Load Forecasting Approach Optimized by Bacterial Colony Chemotaxis Method[J]. Proceeding of the CSEE, 2011, 31(34): 93-99. 